\title{
Review: 14 risk factors for post-traumatic stress disorder include childhood abuse and family psychiatric history
}

\author{
Brewin CR, Andrews B, Valentine JD. Meta-analysis of risk factors for posttraumatic stress disorder in trauma-exposed \\ adults.J Consult Clin Psychol 2000 Oct;68:748-66.
}

QUESTIONS: In adults exposed to trauma, what are the risk factors for post-traumatic stress disorder (PTSD)? Are the effect sizes for risk factors influenced by sample and study characteristics?

\section{Data sources}

Studies were identified by searching the Social Science Citation Index and PILOTS (National Center for PTSD database), recent journal issues, review articles, and book chapters.

\section{Study selection}

English language studies were selected. Exclusion criteria were incomplete assessment of PTSD; participants with less severe post-traumatic symptoms or partial PTSD were included in the same group as those meeting PTSD diagnostic criteria; all participants already had PTSD or another psychiatric disorder; not all participants had been exposed to trauma; or data were insufficient for calculating effect sizes.

\section{Data extraction}

Data were extracted on trauma type, participant characteristics, diagnostic criteria, study design, and predictors. Risk factors assessed in $\geqslant 4$ studies were included in the meta-analysis.

\section{Main results}

77 studies met the selection criteria (sample size range 25-4127 adults). Trauma types were war (28 studies), crime (13 studies), disaster (9 studies), motor vehicle accidents (4 studies), burns (6 studies), other specific traumas (7 studies), and mixed traumas (10 studies). All 14 risk factors predicted PTSD but effect sizes varied (table). Risk factors varied in their homogeneity, with psychiatric history, childhood abuse, and family psychiatric history consistently predicting the disorder, whereas other factors did not predict equally well across studies. The effect of sample and study characteristics on risk factors were assessed: military $v$ civilian samples, men $v$ women, retrospective $v$ prospective designs, analysis by diagnosis $v$ continuous scores, PTSD assessed by interviews $v$ questionnaires, and whether trauma occurred in childhood. The effect sizes for childhood abuse, family psychiatric history, low intelligence, lack of social support, and life stress were not affected by these study and sample characteristics.

\section{Conclusions}

In adults exposed to trauma, risk factors for posttraumatic stress disorder include childhood abuse, family psychiatric history, low intelligence, lack of social support, and life stress. Study or sample characteristics affect some effect sizes.

Risk factors for post-traumatic stress disorder

\begin{tabular}{|c|c|c|c|c|}
\hline Risk factor & $\begin{array}{l}\text { Number of } \\
\text { studies }\end{array}$ & $\begin{array}{l}\text { Number of } \\
\text { participants }\end{array}$ & $\begin{array}{l}\text { Effect size } \\
\text { range } r\end{array}$ & $\begin{array}{l}\text { Weighted } \\
\text { mean } r\end{array}$ \\
\hline Trauma severity & 49 & 13653 & -0.14 to 0.76 & $0.23^{*}$ \\
\hline Lack of education & 29 & 11047 & -0.11 to 0.37 & $0.10^{*}$ \\
\hline Younger age & 29 & 7207 & -0.38 to 0.28 & $0.06^{*}$ \\
\hline Female sex & 25 & 11261 & -0.4 to 0.31 & $0.13^{*}$ \\
\hline Race (minority status) & 22 & 8165 & -0.27 to 0.39 & $0.05^{*}$ \\
\hline Psychiatric history & 22 & 7307 & 0.00 to 0.29 & 0.11 \\
\hline Low socioeconomic status & 18 & 5957 & 0.1 to 0.38 & $0.14^{*}$ \\
\hline Other adverse childhood factors & 14 & 6969 & 0.09 to 0.60 & $0.19^{*}$ \\
\hline Other previous trauma & 14 & 5147 & -0.05 to 0.36 & $0.12^{*}$ \\
\hline Family psychiatric history & 11 & 4792 & 0.07 to 0.28 & 0.13 \\
\hline Lack of social support & 11 & 3276 & -0.02 to 0.54 & $0.40^{*}$ \\
\hline Childhood abuse & 9 & 1746 & 0.07 to 0.30 & 0.14 \\
\hline Life stress & 8 & 2804 & 0.26 to 0.54 & $0.32^{*}$ \\
\hline Low intelligence & 6 & 1149 & 0.08 to 0.38 & $0.18^{*}$ \\
\hline
\end{tabular}

*Heterogeneity among studies was statistically significant.
Source of funding: UK Ministry of Defence Corporate Research Programme.

For correspondence: Dr C R Brewin, Subdepartment of Clinical Health

Psychology, University College London, Gower Street, London

WC1E 6BT, UK. Fax

+44 (0)2079161989.

Abstract and

commentary also appear in

Evidence-Based

Medicine.

\section{COMMENTARY}

At first glance, this important, clearly written, and self critical review by Brewin et al shows that many risk factors contribute to PTSD. This, however, is mostly due to the large number of participants included in each analysis. When effect sizes are considered, a different story emerges, mainly showing that the intensity of the traumatic event and factors that follow exposure ("social support" and "further stressors") are the strongest predictors of PTSD, whereas "previous" (ie, pre-traumatic) factors have smaller individual effects (combined effects were not computed). Thus, once a traumatic event has occurred, the major risk factors for PTSD appear to lie ahead, suggesting ample opportunity for secondary prevention of this disorder. Such a conclusion, however, might have to be reserved for several reasons. Firstly, the proximity of later predictors to the time at which questions were asked could have aggrandised their reported effect. Secondly, this meta-analysis did not address interaction between risk factors. Thirdly, pre-trauma risk factors could affect the survivors' responses to and perception of the traumatic event, and their capacity to respond to rewarding social interaction during the period of recovery. Fourthly, pre-trauma and posttrauma risk factors may have a common source (eg, living environment in which life stressors abound).

Importantly, this work puts the simple causal link between traumatic events and subsequent PTSD into proportion. Trauma exposure is, indeed, a trigger. Preceded by as many vulnerability factors as this work depicts, and followed by potent reinforcing or maintaining factors, the impact of the traumatic event is determined by a chain reaction. Surprisingly, this work did not capture major differences between methods of enquiry (eg, prospective or retrospective; questionnaires or interviews) and populations studied. The differences that were captured wisely show that generalisations are risky and that the relative weight of risk factors should be reconsidered in each case. The role of biological risk factors was not addressed, yet pertinent evidence may become available in the future.

Arieh Y Shalev, MD

Hadassah University Hospital Jerusalem, Israel 